\title{
Ensuring the Spread of Referral Marketing Campaigns: A Quantitative Treatment
}

\author{
Kumar Gaurav ${ }^{1,+}$, Sayantari Ghosh ${ }^{2,+,}$, Saumik Bhattacharya ${ }^{3}$, and Yatindra Nath Singh ${ }^{1}$ \\ ${ }^{1}$ Indian Institute of Technology Kanpur, India \\ ${ }^{2}$ National Institute of Technology Durgapur, India \\ ${ }^{3}$ Indian Institute of Technology Roorkee, India \\ *sayantari.ghosh@phy.nitdgp.ac.in \\ +these authors contributed equally to this work
}

\begin{abstract}
In marketing world, social media is playing a crucial role nowadays. One of the most recent strategies that exploit social contacts for the purpose of marketing, is referral marketing, where a person shares information related to a particular product among his/her social contacts. When this spreading of marketing information goes viral, the diffusion process looks like an epidemic spread. In this work, we perform a systematic study with a goal to device a methodology for using the huge amount of survey data available to understand customer behaviour from a more mathematical and quantitative perspective. We perform an unsupervised natural language processing based analysis of the responses of a recent survey focusing on referral marketing to correlate the customers' psychology with transitional dynamics, and investigate some major determinants that regulate the diffusion of a campaign. In addition to natural language processing for topic modeling, detailed differential equation based analysis and graph theoretical treatment, experiments have been performed for generation of a recommendation network to understand the diffusion dynamics in homogeneous as well as heterogeneous population. A complete mathematical treatment with analysis over real social networks can help us to determine key customer motivations and their impacts on a marketing strategy, which are important to ensure an effective spread of a designed marketing campaign. Pointing out possibilities of extending these studies to game theoretic modeling, we prescribe a new quantitative framework that can find its application to all areas of social dynamics, beyond the field of marketing.
\end{abstract}

\section{Introduction}

Online social networks have become an undeniable accessory in today's life. People habitually use online social networks for conveying information as well as opinion due to the convenience, competence, and substantial dissemination power. Use of social networks is increasing day by day among political campaigners and marketing managers for promoting an idea, product or a brand. If a marketer encourages consumers to share and spread a marketing message through their social contacts, it is called Referral Marketing. As the spread of the message can have an epidemic-like effect, this is also commonly termed as Viral Marketing (VM). Over the past decade, the domain of VM has grown explosively, which now includes passing along advertisements, photos, videos, promotional hyperlinks, animations, games, newsletters, press releases etc. to promote a particular product. In last few years, several studies ${ }^{1-4}$ started to conceptualize VM as a close derivative of disease infection models from mathematical epidemiology. It has been pointed out that understanding the contagion in a population from the perception of a mathematical epidemiologist will be considerably beneficial for the marketers for planning VM campaigns in a more organized and methodical manner.

Model formulation, study and analysis of epidemics have been applied to several problems beyond the boundaries of health and biology over past decade, to successfully depict and understand these phenomena, and device strategies. Starting from the theoretical papers by Kermack and McKendrinck ${ }^{5}$, infectious disease models have been vastly applied to analyse the spread of information, rumor, custom, scientific ideas, opinions, petitions etc ${ }^{6-9}$. In the classical models of epidemiology, the spread of infectious diseases depends mainly on the interactions between susceptible and infected, while the interactions become more complex and nonlinear as the models describe more realistic social scenarios. An epidemic, typically, is defined as a situation in which the number of the infected reaches a significant percentage at steady state. In the case of a VM campaign, it would be a situation where the sharing via social network creates enough momentum so that the marketing message reaches and attracts a majority of its target consumers. In this particular context, purpose of the study will be focused on maximizing the spread, while the usual epidemiology studies aim to contain the epidemic, which in itself adds an interesting perspective to the problem. The spread of marketing messages in social networks raises various theoretical and practical questions: How can an advertisement reach maximum audience? Beyond the design and content, are there any factors that affect this dynamics? What 


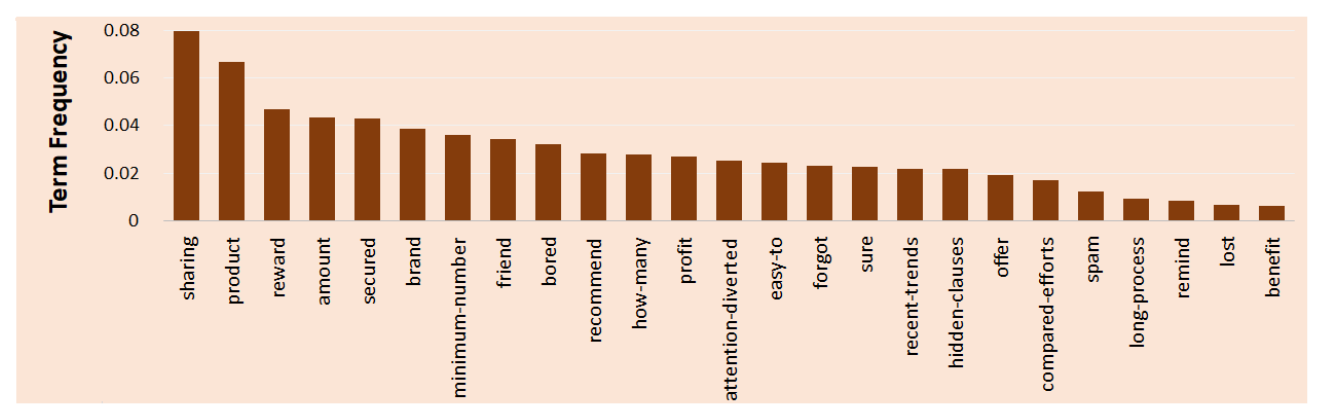

(a)

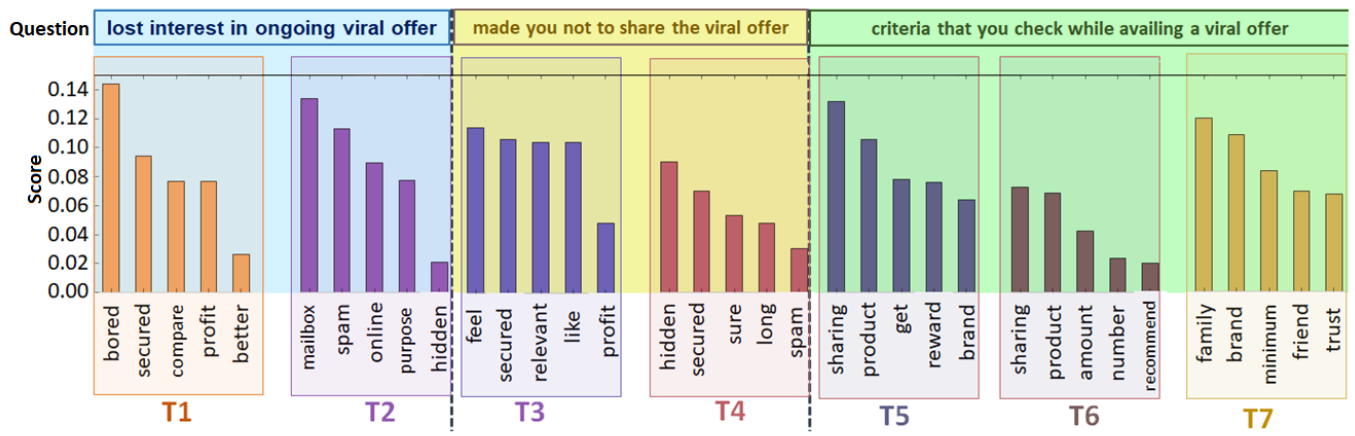

(b)

Figure 1. Analyses of survey data: (a) Top 25 words with highest term- frequency in the open ended and semi-open ended responses after preprocessing as described in Sec. 2.1; (b) topic modeling of processed response. The 7 detected topics (T1-T7) from 3 different open ended questions are shown here. For better visualization, we show 5 words with highest scores in each topic.

actions can the companies take to speed up the diffusion rate?

In marketing, Bass ${ }^{10}$ used underlying epidemic model as a foundation for his new product diffusion model. But, in the context of online social networks and digital contagion, Sohn et al. ${ }^{11}$ first demonstrated the VM diffusion as SIR and SEIAR processes of epidemiology in very recent times. Rodrigues et al. ${ }^{12}$ have proposed a mathematical model of the VM progression, using insights from epidemiology with quantitative treatment. Bhattacharya et al. ${ }^{13}$ demonstrated a more realistic model of VM propagation, where some essential feedback interactions were considered for the first time. However, these models ${ }^{11-13}$ do not reflect the spreading process adequately. Though there are several survey-based studies and marketing data available for VM diffusion which clearly indicate the complex and nonlinear interaction in a population, most of these studies stick to the classical epidemiology models to depict and understand the scenario. An extensive survey by Ghosh et al. ${ }^{14}$ has revealed that beyond the viral components from creative perspective, a clear insight of customer behaviour becomes indispensable for ensuring the relevance and survival of a newly-launched campaign. With the goal to develop a model which is more realistic and data-driven, they implemented the major observations of the survey to create a conceptual framework for diffusion of VM advertisement in society.

In our present study, we analyze the data collected from recent surveys ${ }^{14-17}$, focusing mostly on qualitative studies ${ }^{18}$, which gives a room for introduction of new and complex interactions, as well as conceptual frameworks. First, using Natural Language Processing (NLP) tools and topic modeling, we figure out several important dynamical aspects which need to be incorporated into the model to describe the dynamics more appropriately. As topic modeling can find out the important as well as latent topics in a response, which are difficult to visualize otherwise, we use Latent Dirichlet allocation (LDA) to analyze the survey responses. Next, we propose a flow chart of the dynamics which aligns much better with reality and develop a holistic model for epidemic-like spreading of VM campaigns. Furthermore, the implications of spams and brand names on consumer attitude and the process of an inert becoming a Broadcaster is analyzed more deeply. To understand the VM dynamics, mean-field equations are derived and numerical simulations are carried out. The dynamics have also been studied keeping heterogeneity of social systems in mind and extensive simulation based studies were carried out over random, scale-free and real social networks. Due to the unavailability of an existing recommendation network structure in public domain, an experiment has been carried out to generate the structure of a recommendation network and to study the dynamics on recommendation-based contact network. 


\section{Quantitative and Qualitative Analyses of Survey Data}

\subsection{Detecting Important Words and Topics}

To understand the sentiments of customers, we extensively analyze the collected survey results of Ghosh et al. ${ }^{14}$ using different language processing techniques which are explained in Sec. 5 (Methods). The analysis has been done on the responses of the open ended questions, i.e., where users were asked to explain/ justify certain answers, and semi-open ended questions, i.e., where users can add answers other than the choices provided with the respective questions. A series of data pre-processings are applied on the collected data to capture the user-sentiments and the probable factors that were not considered by the surveyors while circulating the survey. First, we analyze the responses using term frequency (TF) analysis ${ }^{19}$. This helps us to observe the words that are occurring frequently in the responses due to their importance. However, the computation of term frequency requires a careful filtering to remove commonly occurring words like 'a', 'the', 'is' etc., as these words would otherwise shadow the important words related to the specific survey. We also group the synonymous words, e.g. \{reward, benefit, prize $\}$, \{spam, bulk, phishing \} etc., so that the cumulative true impact of these factors can be observed. The results of the term frequency analysis is shown in Fig. 1 (a), which demonstrates that words like 'sharing', 'product', 'brand', 'secure', 'bored', 'hidden' etc. are used frequently by the participants to establish their viewpoints while answering the questions. The most frequently occurring words contain both 'positive' words, i.e., the factors that increases someone's probability to be engaged in viral campaigning activity, and 'negative' words, i.e., the factors that decreases the probability of becoming an active broadcaster of a viral message. 'Rewards', 'easy', 'secured' are some of the frequently occurring positive words, whereas 'bored', spam', 'hidden clauses', 'long process' etc. are some negative words. There are also some frequently appearing words in TF which are not easily interpretable, e.g. 'recent trends','friend', 'share' etc. Thus, even after manual preprocessing of the data, not all the latent sentiments are clear from the survey responses using TF analysis. To better understand the correlation between these responses and the sentiments of the users we also perform topic modeling of the data using classical Latent Dirichlet allocation (LDA $)^{20}$ to find out the topics in the data. LDA is an unsupervised generative probabilistic method which can capture the underlying broad topics in a document. Assuming a response as mixture of different sentiments and factors that control the engagement of an individual in a VM campaign, we performed the LDA analysis on the same preprocessed data. In case of LDA, the preprocessing helps to generate a bag of exclusive words, rather than a simple bag of words. The LDA clearly exhibits that for decision making, the participants considered different factors like content (T1 in Fig. 1(b)) security (T3 and T4 in Fig. 1(b)), amount of profit (T5 in Fig. 1(b)), ease of sharing (T6 in Fig. 1(b)) and personal trust on the recommender as well as on the brand (T7 in Fig. 1) to forward or reject a received viral offer. To propose our model, we critically analyze these topic modeled open-ended and close-ended responses.

\subsection{Important factors driving customer motivation}

In the diffusion process of a VM campaign, there are three obvious states in which a member of the population could be in: unaware of the message; received (and passing along) the message; and aware, but inactive about the message. The natural course that a campaign follows, is to pass-through these three sub-populations. For a successful campaign, a definite percentage of the population remains active through-out this diffusion, and even after equilibrium is attained. Beyond this simple linear flow of the campaign, there are also several more complex interactions that are very much probable on a social network. For example, in Fig. 1(b), T7 indicates that friends and family has certain influence in someone's participation in a campaign. To understand these driving factors in referral marketing, we start by reporting our observations about some major factors which reflect customer's perspective about marketing messages. These observations are according to the outputs of the LDA analysis which are co-aligned with the findings of several recent surveys ${ }^{14,21}$.

\subsubsection{Inherent Aversion: Rigidly Inert}

The marketing platform that takes e-mail forwarding as one of the key methods to spread word-of-mouth nowadays, suffers strongly due to spam e-mails. VM is essentially attached with emailing (or electronically sending) a company constructed promotional statement to acquaintances, which tends to be perceived as spam as soon as the required number of sharing starts growing. From the analysis of survey data ${ }^{14}$ in Sec. 2.1 , we note that the aversion towards bulk messages, possibly with fake commitments, has constructed the major theme of T2 and T4 in Fig. 1(b). Our TF analysis also supports this claim; from Fig. 1 (a), an important observation to point out is that the repulsion of users towards spam mails came out as an emergent theme from the spontaneous responses, while the interviewer never mentioned this idea in the questions. According to our results, the respondents have clearly associated their reasons for losing interest or not participating in a referral request with words like 'spam', 'hidden' and '(not) secured'. Support to our LDA study can also be provided from several survey-based studies where the participants clearly mentioned that they often confuse the marketing mails with the spams. To mention a few, in a study by Raad et al. ${ }^{15}$, they observed that to avoid the risk of the spam, a majority of participants were not willing to take the risk of even opening the marketing e-mails. Phelps et al. ${ }^{22}$ have clearly pointed out that boundary between spam and pass-along marketing mails is not always clear to the recipients. Several studies ${ }^{17,23,24}$ have shown that many recipients 


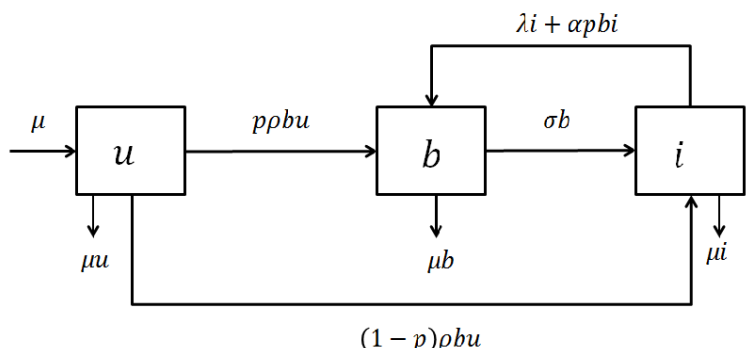

Figure 2. A schematic diagram of the proposed model. The arrows along with the parameters indicate the possible transitions from one state to other and the rate of transitions respectively.

associate a feeling of intrusion, irritation, invasion of privacy and general security concerns with unsolicited marketing e-mails and mobile messages. Thus, based on our observation, we conclude that, for a realistic modeling, a class among participants must be considered who are strongly against bulk, unsolicited mailing, and thus, referral marketing, in general.

\subsubsection{Brand Trust}

In marketing campaign propagation, the brand name plays a crucial role ${ }^{25-28}$. People comfortably share marketing messages from popular brands which can attract substantial numbers of prospective consumers. Research studies ${ }^{29,30}$ have correctly pointed out that in this era of seamless social persona, people understand the necessity to stand behind their words, and thus they inherently tend to believe reputed brands from the aspects of privacy and authentic engagement. Interestingly, two consecutive surveys in 2015 and $2017^{31}$ pointed out, that customers identify a brand as unprofessional, suspect superficiality, and finally email opt-out (or unsubscribe) in case of excessive frequency of promotional mails $\mathrm{s}^{32}$, which are perceived as spams. Analysing the survey that we focus on ${ }^{14}$, we also found that the idea of spam and brands to be closely related. In response to direct questions, 39\% respondents thought that recognized brands will not be associated with spamming, contextually blind automated bulk mailing and falsifying brand stories. Through further analysis of survey data in Sec. 2.1, we also detect that the topic T7 (Fig. 1(b)) associates the word 'brand', with words like 'family', 'friend' and 'trust'. This shows that people need to 'trust' the person who is referring the product (e.g., 'family' or 'friend') as well as the brand-names while participating in referral marketing. We consider this as an important observation which should be incorporated in the model of campaign diffusion, by introducing a parameter that will take care of the brand reputation and popularity in the mass. This will directly contribute in making people interested about the campaign in the first place, if the brand is trustworthy, and will also nudge them to get back to the product, if they become inert eventually.

\subsubsection{Remembering and Reminding}

Another important aspect that we notice in the survey ${ }^{14}$ is the distinctive reasons that can make a person inert about an advertisement campaign. While losing interest after being active for some time could be one way, for many customers simply forgetting about the message was the case as well. Thus in TF analysis (Fig. 1(a)), we have high values for the words 'bored', 'attention-diverted' and 'forgot'. People who lost interest, got bored or doubtful (due to low profit-to-effort ratio) while broadcasting the campaign are easily intrigued by an authentic success story of the product/campaign. Studies have shown that a genuine information highlighting a substantial reward from a particular campaign can turn them to active participants ${ }^{14,21}$. On the other hand, people who forgot or got diverted for some reason were usually, in the first place, interested to avail the offer. There could be several induced ways to bring them back to the active group as they will not need much persuasion.

- Strategically designed retargeting emails from the company ${ }^{33}$ or encountering catchy slogans that tap into the visceral states to motivate immediate consumer behavior ${ }^{34}$ could be one of the most successful ways to motivate them to return to the active state. Here they are not influenced by another individual, but by the company itself, and here the latent interest of the customer is essential.

- Casual reminders from friends ${ }^{22}$ or participation in recent discussions about a particular product in their own social circle $^{35}$ (commonly termed as buzz) can tempt the inerts to become active again. This happens due to direct influence of peers which might need some persuasion. As shown in topic T3 (Fig. 1(b)), relevance of the campaign is an important aspects to the users. This relevance can be reminded or discussed by family members or friends. In TF analysis (Fig. 1(a)) also, we observe higher frequencies of the terms like 'remind', 'recommend', 'friends', 'recent-trends' etc. The brand name can also play a crucial role here as people avoid sending provocative marketing messages from lesser known advertisers to friends repetitively ${ }^{16,28}$ 


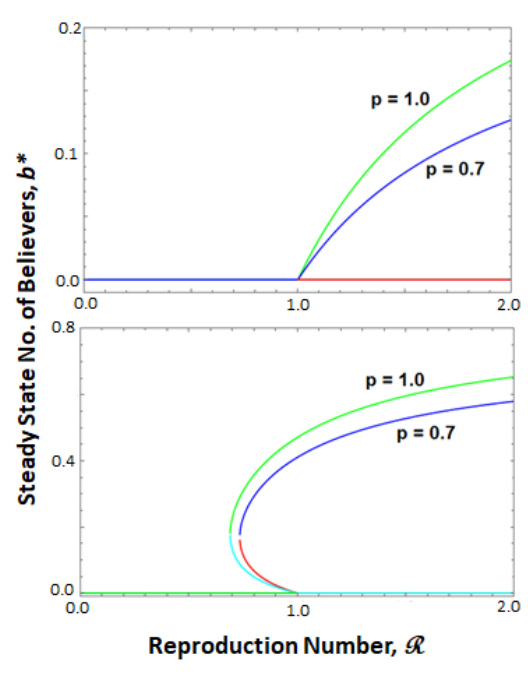

(a)

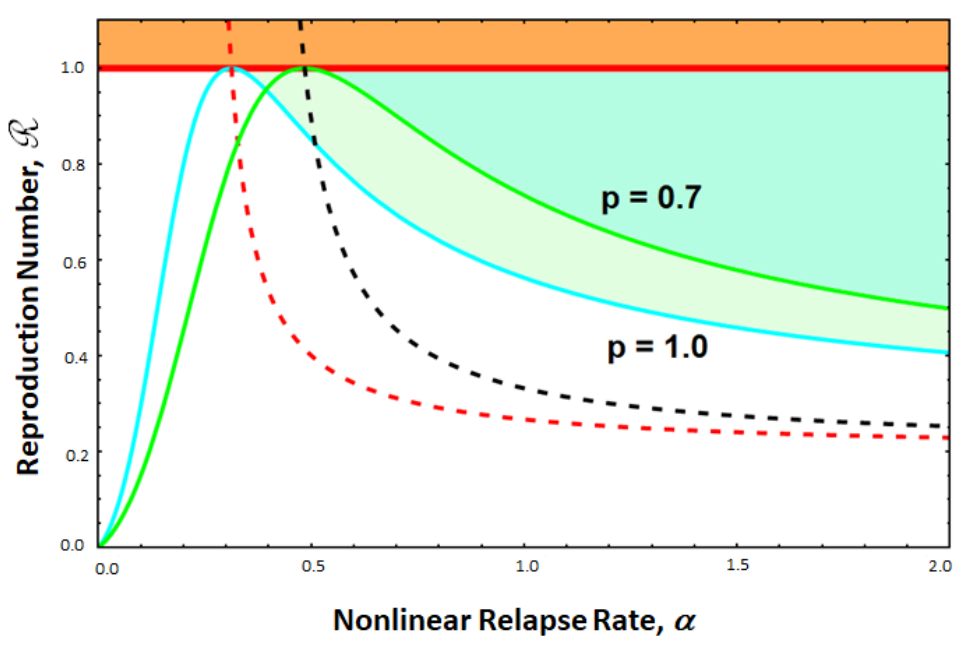

(b)

Figure 3. (a) Variation in steady state fraction of $b$ with reproduction number $\mathscr{R}$ for (upper panel) $\alpha=0.1$, when only a single epidemic state persists beyond $\mathscr{R}=1$ and for (lower panel) $\alpha=1$, when bistability can be observed in range $\mathscr{R}_{c}$ to 1 .

Parameter values are $\sigma=0.2, \lambda=0.02$, and $\mu=0.05$. In these figures, green (and blue) lines indicate stable solutions while cyan (and red) lines indicate unstable solutions for $p=1$ (and $p=0.7$ respectively). For these parameter values, we calculated $\mathscr{R}_{c}=0.562$ (and $\mathscr{R}_{c}=0.594$ ) for $p=1$ (and $p=0.7$ respectively) using Eq. 6. (b) Phase diagram of the model in $\alpha-\mathscr{R}$ space for $\sigma=0.2, \lambda=0.02$ and $\mu=0.05$. The red line indicates $\mathscr{R}=1$. The region filled with orange color beyond $\mathscr{R}=1$ always exhibits monostable endemic state. The cyan (and green) line indicates $\mathscr{R}_{c}$, while the red (and black) dashed line indicates $\alpha_{t h}$ for $p=1.0$ (and $p=0.7$ ). The white region, where $\alpha<\alpha_{t h}$, exhibits monostable VM free state. In the green shaded region for $p=1.0$ (and darker green shaded region for $p=0.7$ ), $\alpha>\alpha_{t h}, \mathscr{R}>\mathscr{R}_{c}$ and $\mathscr{R}<1$. Thus, this area exhibits bistability, where either VM free state or the endemic state is chosen by the system depending upon the initial state.

So, while modeling the diffusion process mathematically, we must consider the complexity of these relapses.

\section{Data-driven Model: Formulation and Results}

\subsection{Formulation of Model based on Data Analyses}

Analyzing the survey and the interaction between different people, we model the transition between sub-groups under the influence of the above mentioned factors in Fig. 2. To study the mean-field behavior, the total population $(T)$ is divided into three non-overlapping subpopulations : Unaware $(U)$ class who are yet to receive the message or the campaign, Broadcaster $(B)$ class who have the potential to spread the campaign and Inert (I) class who are willingly or unwillingly not taking part in the campaign though they have come across it at least once. The unaware class $U$ contains the target market or the susceptible people, who have not received the message yet but have a chance of receiving it from the broadcasters. In the broadcaster class $B$, we consider the individuals who has received the message earlier and have the potential to spread the message further to their contacts. If a member in the broadcaster spreads the message by recommending it through his social contacts, we consider the member is in active state. We assume that at a rate $\rho$, a broadcaster spreads the message to a member from unaware class, which creates new prospective broadcasters. We consider that whenever a broadcaster sends the referral message to an unaware individual, unaware moves to broadcaster class with probability $p$ and to inert class with probability $(1-p)$. We denote $p$ as the trust parameter, which assumes a high value if the campaign is from a trusted brand or the message comes from a trusted member. The impact, or acceptability of the campaign to the unaware community is accounted using this probability parameter, $p$. Based on our discussions in Sec. 2.2.1, the term $(1-p)$ takes into account of the fact that some of the people from unaware class might have an inherent aversion to spam-like messages, and decide to ignore it straightaway. Messages from not so trustworthy brand or members increases the value of $(1-p)$.

For various reasons people who used to be in broadcaster class $B$, can stop sharing the message, and move to the inert class $I$. As we have seen in Sec. 2.2.3, there are two distinctive ways to bring these inert people back to broadcaster class: the first one 
could be independent of their interaction with another individual (like, reminder from the company etc.), while the second one is induced by their interaction with another broadcaster, directly or indirectly (like, reminder from a friend, discussion with family member). To justify all these possible transitions from $I$ to $B$, we have included two feedbacks from inert class to broadcaster: one, a linear transition from inert to broadcaster with rate $\lambda i$, and another, a nonlinear interaction-driven transition. Considering the observations discussed in Sec. 2.2, we decide that nonlinear relapse should depend on how well-known the brand is, and thus, we incorporate the probability $p$, the brand-trust parameter, in this relapse rate, considering the term as $\alpha^{\prime} b i$, while $\alpha^{\prime}=\alpha p$, where $\alpha$ is the original relapse rate, and $p$ again takes care of the acceptability of the advertisement to a person. In practical scenarios, people enter and leave the population. To include this factor, we have introduced birth and death in our model. Both birth and death rates are kept equal to $\mu$, so that a fixed population size can be maintained ${ }^{12}$. For a particular VM dynamics, birth and death can be viewed as events when people join or leave a particular social platform where the campaign is going on. Considering numbers of unaware, broadcaster and inert individuals as continuously varying quantities, switching between subpopulations can be modelled by the following set of coupled ordinary differential equations:

$$
\begin{aligned}
u^{\prime} & =\mu-\rho b u-\mu u \\
b^{\prime} & =p \rho b u+\lambda i+\alpha^{\prime} b i-\sigma b-\mu b \\
i^{\prime} & =\sigma b+(1-p) \rho b u-\lambda i-\alpha^{\prime} b i-\mu i
\end{aligned}
$$

where, $\alpha^{\prime}=\alpha p$, and the rest of the parameters have significance as depicted in Fig. 2.

\subsection{Mean Field Study}

\subsubsection{Reproduction Number}

Reproduction number for a marketing message propagation can be defined as the expected number of secondary broadcasters produced by typically a single person, who is recommending a product to his peers in a completely susceptible population. For simplistic models of mathematical epidemiology, this quantity defines the epidemic threshold of a particular infection. As found in the Sec. 5.2, reproduction number of the model is

$$
\mathscr{R}=\frac{\rho(\lambda+\mu p)}{\mu(\lambda+\mu+\sigma)}
$$

From Eq. 2, we can observe that, for smaller value of $p$, a larger value of $\rho$ is required to satisfy the basic condition for an epidemic to spread, i.e., $\mathscr{R}>1$. In a practical sense, it means that if more people are switching directly to inert class from unaware class, may be for a lesser known brand, more marketing effort will be required to attain an endemic steady state. The sensitivities of $\mathscr{R}$ for various parameters are as follows:

$$
\begin{gathered}
\Gamma_{\rho}^{\mathscr{R}}=1 ; \quad \Gamma_{p}^{\mathscr{R}}=\mu p ; \quad \Gamma_{\sigma}^{\mathscr{R}}=-\frac{\sigma}{(\lambda+\mu+\sigma)} ; \quad \Gamma_{\lambda}^{\mathscr{R}}=\frac{\lambda}{(\lambda+\mu p)} \frac{\sigma+\mu(1-p)}{(\lambda+\mu+\sigma)} ; \\
\Gamma_{\mu}^{\mathscr{R}}=-\left(\frac{\lambda}{\lambda+\mu p}+\frac{\mu}{\lambda+\mu+\sigma}\right) .
\end{gathered}
$$

It can be observed that $\mathscr{R}$ changes linearly with $\rho$. Thus, in our experiments, we change $\rho$ to vary $\mathscr{R}$ keeping other parameters fixed to analyze system dynamics and bifurcation. Positive value of $\Gamma_{p}^{\mathscr{R}}$ also gives us the indication that brand reputation plays an important role in the final steady state values.

\subsubsection{Bifurcation \& its significance}

The relapse rate happens to be a very important factor that defines the nature of the dynamics, as the model exhibits different behavior for smaller and larger value of $\alpha$ (discussed in Sec. 5.2). In Fig. 3(a), we plot the steady-state fraction of class $B$ for two different values of $\alpha$. To highlight the impact of the parameter $p$, results have been shown for $p=1$ and $p=0.7$. The upper panel of Fig. 3(a) shows the results for $\alpha=0.1$, a smaller relapse rate. This depicts that though the epidemic occurs after $\mathscr{R}_{c}=1$, the endemic steady-state fraction of broadcasters, $b^{\star}$, reduces as the value of $p$ decreases. For a smaller value of $p$, the probability of an unaware individual to move to broadcaster class will be less, and more people will be moving from unaware class to inert class directly. As the relapse rate, $\alpha$ is also small and it is scaled with $p$ as well, switching from inert to broadcaster will take place at a smaller rate. Reduction in broadcaster fraction, $b^{\star}$ can be attributed to the combined effect of these two parameters. It shows the effect due to an less appealing or less trusted advertisement campaign (smaller $p$ ), where no substantial efforts have been invested in ensuring relapse of lost customers ( $\operatorname{smaller} \alpha$ ).

In the lower panel of Fig. 3 (a), we can see that for a higher value of $\alpha$, a drastically different behavior can be observed. In this case, the significant change can be detected in the region $\mathscr{R}<1$, where we can see the emergence of a region of bistability. It clearly demonstrates that an endemic state, where prevalence of the viral campaign in ensured in the population, can even occur for $\mathscr{R}<1$ within a critical value of $\mathscr{R}=\mathscr{R}_{c}$. Even when $p$ decreases bistability still persists, suggesting that sustainability of the campaign can even be achieved for less appealing advertisements, iff, a significantly high relapse or regaining of inert 
customers can be accomplished. For any $p<1$, although a fraction of unaware population is coming directly to the inert class, the large value of $\alpha$ brings them back to the broadcaster class establishing endemic state till $\mathscr{R}=\mathscr{R}_{c}$, which is markedly less than 1 . We will proceed to figure out the expression of $\mathscr{R}_{c}$ in the next section.

\subsubsection{Conditions for Bistability}

Bistability observed in our model system implies dependence of system dynamics on history (i.e., initial state of the population), which is commonly termed as hysteresis ${ }^{36}$. We have shown in Sec. 5.2 that bistable region (multiple stable steady state solutions) may exist when $\mathscr{R}<1$, for larger values of $\alpha$. There are two conditions that must be satisfied to ensure bistability through the coefficients in Eq. 14.

- Limiting value of induced relapse, $\alpha$ : Equating the coefficient $m$ of Eq. 14 to zero, we found threshold value of $\alpha$

$$
\alpha_{t h}=\frac{\rho(\sigma+\mu+\lambda)}{p(\rho-\mu)} \text {. }
$$

- Limiting value of infectious contact, $\rho$ : Again from Eq. 14, the critical value of $\rho$ can be calculated by equating $m^{2}-4 \ln$ to zero and is given by

$$
\rho_{c}=\frac{\alpha \mu}{(\alpha+\lambda+\mu+\sigma)-2 \sqrt{\alpha(\mu-p \mu+\sigma)}} .
$$

Substituting $\rho_{c}$ in place of $\rho$ in the expression of reproduction number $\mathscr{R}$ (Eq. 2) gives the critical value of reproduction number $\mathscr{R}_{c}$ as

$$
\mathscr{R}_{c}=\frac{\rho_{c}(\lambda+\mu p)}{\mu(\lambda+\mu+\sigma)}=\frac{\alpha \mu}{(\alpha+\lambda+\mu+\sigma)-2 \sqrt{\alpha(\mu-p \mu+\sigma)}} \frac{(\lambda+\mu p)}{\mu(\lambda+\mu+\sigma)} .
$$

Fig. 3(b) depicts the dynamical behaviour in form of a phase diagram in $\alpha-\mathscr{R}$ space for $\sigma=0.2, \lambda=0.02$ and $\mu=0.05$. We point out that the region of bistability here indicates the sustainability of the advertisement campaign in a population even when $\mathscr{R}<1$, i.e., the infective contacts are not that effective. We note that the region of bistability shrinks as $p$, the brand-trust parameter decreases. It clearly points out that well-known brands are much more prone to have resonant stories in social media due to their established brand-value.

\subsection{Graph-theoretical Analysis}

\subsubsection{Model Dynamics on Networks}

In contrast to mean-field approach, diffusion in networks will be dependent on the degree distribution of the network. We denote with $u_{k}, b_{k}$, and $i_{k}$ the fraction of unaware, broadcaster and inert nodes with degree $k$. Nodes having same degree are considered to behave in same fashion. Using this degree block approximation, differential equations for evolution of degree based compartments of different class will be

$$
\begin{aligned}
u_{k}^{\prime} & =\mu-\rho_{n} k u_{k} \Theta_{b}-\mu u_{k} \\
b_{k}^{\prime} & =p \rho_{n} k u_{k} \Theta_{b}+\lambda i_{k}+\alpha_{n} k p i_{k} \Theta_{b}-(\sigma+\mu) b_{k} \\
i_{k}^{\prime} & =\sigma b_{k}+(1-p) \rho_{n} k u_{k} \Theta_{b}-\lambda i_{k}-\alpha_{n} k p i_{k} \Theta_{b}-\mu i_{k}
\end{aligned}
$$

We are considering $\rho_{n}$ to be the rate at which a broadcaster spreads the information to an unaware neighbor. Similarly, $\alpha_{n}$ is the relapse rate influenced by the neighbors. Subscript ' $n$ ' in both these symbols signify network setting and they are counterpart of $\rho$ and $\alpha$ used in homogeneous setting. $\Theta_{b}$ is the density function which gives probability of broadcasters around a node. In a general scenario, density function of any class around a node depends on degree of the node but for uncorrelated network, it is independent of degree $k$ and is given by $\Theta_{b}=\sum_{k} \frac{k p_{k} b_{k}}{\langle k\rangle}$.

Multiplying all three equations of Eq. 7 by $\frac{k p_{k}}{\langle k\rangle}$ and then performing summation over $k$, we get

$$
\begin{aligned}
& \Theta_{u}^{\prime}=\sum_{k} \frac{k p_{k}}{\langle k\rangle} \mu-\rho_{n} \sum_{k} \frac{k^{2} p_{k}}{\langle k\rangle} u_{k} \Theta_{b}-\mu \sum_{k} \frac{k p_{k}}{\langle k\rangle} u_{k} \\
& \Theta_{b}^{\prime}=p \rho_{n} \sum_{k} \frac{k^{2} p_{k}}{\langle k\rangle} u_{k} \Theta_{b}+\lambda \sum_{k} \frac{k p_{k}}{\langle k\rangle} i_{k}+\alpha_{n} p \sum_{k} \frac{k^{2} p_{k}}{\langle k\rangle} i_{k} \Theta_{b}-(\sigma+\mu) \sum_{k} \frac{k p_{k}}{\langle k\rangle} b_{k} \\
& \Theta_{i}^{\prime}=\sigma \sum_{k} \frac{k p_{k}}{\langle k\rangle} b_{k}+(1-p) \rho_{n} \sum_{k} \frac{k^{2} p_{k}}{\langle k\rangle} u_{k} \Theta_{b}-\lambda \sum_{k} \frac{k p_{k}}{\langle k\rangle} i_{k}-\alpha_{n} p \sum_{k} \frac{k^{2} p_{k}}{\langle k\rangle} i_{k} \Theta_{b}-\mu \sum_{k} \frac{k p_{k}}{\langle k\rangle} i_{k}
\end{aligned}
$$




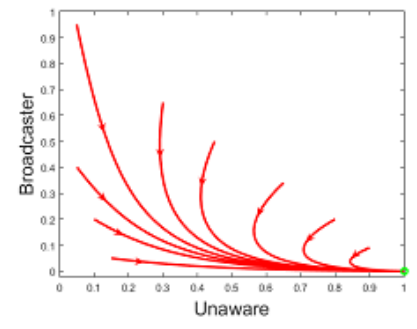

(a)

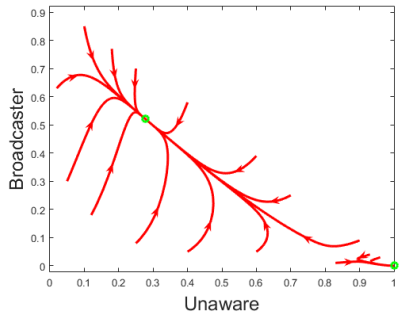

(b)

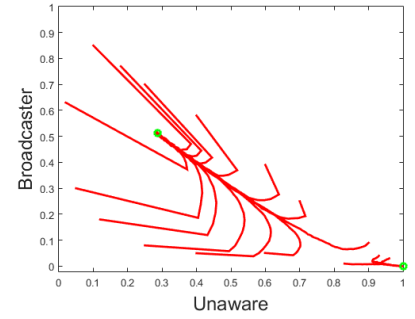

(c)

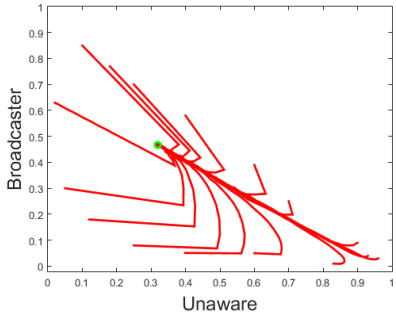

(d)

Figure 4. Numerical simulation of convergence to the steady state for different initial conditions with parameter values $\mu=$ $0.05, \rho=0.25, \sigma=0.2, \lambda=0.02, p=0.7$, and (a) $\alpha=0.1$ for a homogeneous system with a single campaign free steady state; (b) $\alpha=1$ for a homogeneous system with bistable steady states ; Temporal variation of $u$ and $b$ with different initial conditions for equivalent parameter regime as for (b) in (c) random network and (d) scale-free network. In all of these figures, $\mathrm{X}$ and $\mathrm{Y}$ coordinates of the initial point of any flow represents the initial fractional population of unaware and broadcaster class of the population.

We solve Eq. 8 at initial phase of the campaign and at the steady state to get the epidemiological threshold for the heterogeneous structure.

\subsubsection{Propagation at Initial State}

Solving simultaneous linear differential equations Eq. 17 and Eq. 18, we have

$$
\frac{\mathrm{d}^{2} \Theta_{b}}{\mathrm{~d} t^{2}}-\left(C_{1}+C_{4}\right) \frac{\mathrm{d} \Theta_{b}}{\mathrm{~d} t}+\left(C_{1} C_{4}-C_{2} C_{3}\right) \Theta_{b}=0
$$

As clear from the form of the equation, $\Theta_{b}$ will be the summation of two exponential, exponent of which depends on roots of auxiliary equation of the differential Eq. 9. For campaign to spread, $\Theta_{b}$ needs to be an increasing function in time which in turn needs both roots of the auxiliary equation to be positive. So, required condition is $C_{1} C_{4}>C_{2} C_{3}$. Substituting expression of all these constant terms we have the condition

$$
\frac{\rho_{n}}{\mu}\left(\frac{\lambda+p \mu}{\mu(\sigma+\lambda+\mu)}<\frac{\langle k\rangle}{\left\langle k^{2}\right\rangle}\right.
$$

Replacing $\rho_{n}$ by $\frac{\rho}{\langle k\rangle}$ the condition modifies to

$$
\frac{\rho}{\mu} \frac{(\lambda+p \mu)}{(\sigma+\lambda+\mu)}=\mathscr{R}>\frac{\langle k\rangle^{2}}{\left\langle k^{2}\right\rangle}
$$

Left-hand side of the inequality is the reproduction number $\mathscr{R}$ of the homogeneous model and right-hand side is $\frac{\langle k\rangle^{2}}{\left\langle k^{2}\right\rangle}$ which depends on average degree and average of square of individual degrees of the nodes in the network. The exact value of the expression will depend on the type of network. The expectation of $k^{2}$ for a random network with Poisson degree distribution is $\left\langle k^{2}\right\rangle=\langle k\rangle(\langle k\rangle+1)$.

Using the expression for $\left\langle k^{2}\right\rangle$ in Eq. 11 , we get $\mathscr{R}>\frac{\langle k\rangle}{\langle k\rangle+1}$ which can be further approximated by $\mathscr{R}>1$ if $\langle k\rangle>>1$. It is important to observe that from the mean field analysis, we achieved the same condition of epidemiological outbreak. If we consider a scale-free network, its degree distribution can be written as $p(k)=B k^{-\gamma}$. Using this degree distribution, we estimate the average degree as $\langle k\rangle=B \frac{1}{\gamma} l^{2-\gamma}$, and average of degree square as $\left\langle k^{2}\right\rangle \approx B \int_{l}^{\infty} k^{2-\gamma} d k$. When $\gamma \in(2,3] ;(2-\gamma)$ is in range of $[-1,0) ;\left\langle k^{2}\right\rangle$ diverges, and for $\gamma>3,\left\langle k^{2}\right\rangle$ is finite. In other words, in the range $\gamma \in(2,3]$, there is no threshold for epidemiological outbreak. For other values of $\gamma$, we will observe similar behaviour like random network with different diffusion rate.

\subsubsection{Steady State Network Analysis}

As discussed in Sec. 5.5, in steady state, the expression of $\Theta_{b}$ becomes a consistency equation, i.e., we have $\Theta_{b}=f\left(\Theta_{b}\right)$. At $\Theta_{b}=0, f\left(\Theta_{b}\right)$ is also zero. Hence $\Theta_{b}=0$ is a solution of the equation. Value of the function at $\Theta_{b}=1$ is

$$
f(1)=\frac{1}{\langle k\rangle} \sum_{k} \frac{p_{k} k^{2} \rho_{n}\left(\mu p+\lambda+\alpha_{n} k p\right)}{\left(\mu+\rho_{n} k\right)\left(\lambda+\mu+\sigma+\alpha_{n} k p\right)}=\frac{1}{\langle k\rangle} \sum_{k} \frac{p_{k} k}{\left(1+\frac{\mu}{\rho_{n} k}\right)\left(1+\frac{\sigma+(1-p) \mu}{\lambda+\mu p+\alpha_{n} k p}\right)}
$$


Table 1. Important characteristics of different network

\begin{tabular}{|c|l|l|l|l|}
\hline $\begin{array}{c}\text { Network } \\
\text { characteristics }\end{array}$ & $\begin{array}{c}\text { Hamster } \\
\text { Network }\end{array}$ & $\begin{array}{c}\text { Email } \\
\text { Network }\end{array}$ & $\begin{array}{c}\text { Jazz } \\
\text { Network }\end{array}$ & $\begin{array}{c}\text { Recommendation } \\
\text { Network }\end{array}$ \\
\hline Number of nodes & 2426 & 1133 & 198 & 1157 \\
\hline Number of edges & 16631 & 5451 & 2742 & 2558 \\
\hline Average degree & 13.71 & 9.624 & 27.7 & 4.422 \\
\hline Maximum degree & 273 & 71 & 100 & 59 \\
\hline Power law Exponent & 2.46 & 6.77 & 5.27 & 1.34 \\
\hline
\end{tabular}

It is clear from the above expression that $f(1)<1$. To have another solution in the interval 0 to 1 , slope of the function at $\Theta_{b}=0$ must be greater than 1.

$$
\left.\frac{\mathrm{d} f\left(\Theta_{b}\right)}{\mathrm{d} \Theta_{b}}\right|_{\left(\Theta_{b}=0\right)}=\frac{1}{\langle k\rangle} \sum_{k} \frac{p_{k} k^{2} \rho_{n}(\mu p+\lambda)}{\mu(\lambda+\mu+\sigma)}=\frac{\rho_{n}(\mu p+\lambda)}{\mu(\lambda+\mu+\sigma)} \frac{\left\langle k^{2}\right\rangle}{\langle k\rangle} \geq 1
$$

After replacing $\rho_{n}$ by $\frac{\rho}{\langle k\rangle}$, we will get the same condition what we had in early stage analysis, i.e., $\frac{\rho}{\mu} \frac{(\lambda+p \mu)}{(\sigma+\lambda+\mu)}=\mathscr{R}>\frac{\langle k\rangle^{2}}{\left\langle k^{2}\right\rangle}$.

\subsection{Numerical Results}

We have carried out the simulations for homogeneous as well as heterogeneous approach. Along with random and scale free networks, some real network structures have also been considered, including one constructed from our own referral experiment. To compare the results of deterministic mean-field model with network model, we select same set of parameters values in simulations.

\subsubsection{Simulation of Deterministic Model}

Depending upon the parameter values, system may lead to message-free state or endemic state. Two different cases for homogeneous setting have been shown in Fig 4(a)-(b). As discussed for Eq. 4, bistability can be observed in the system for value of $\alpha$ greater than $\alpha_{t h}$. It is observed in Fig. 4 (b), depending on initial fraction of different classes, system reaches to endemic or message-free equilibrium; this exhibits hysteresis.

\subsubsection{Simulation over Model Networks}

Parameter values for bistable case have been used to plot the results for both of the model networks, random as well as scale-free network in Fig. 4 (c) and (d) respectively. Results of random network almost matches the findings of homogeneous model. Along with similar endemic steady-state values, bistability can also be observed in random network scenario of Fig. 4 (c). In case of scale-free network, endemic steady state values are not exactly same and maximum error in endemic steady-state fraction of a particular class is $5 \%$. Under bistable parameter set, message-free steady state never appears and system leads to endemic steady state for every set of initial conditions. It can be observed in Fig. 4(d) where every flow terminates at endemic steady state. This observation is in alignment with our analytic result regarding absence of epidemic threshold in scale-free network as mentioned in Sec. 5.4.

Degree-wise steady state fraction $u_{k}, b_{k}$, and $i_{k}$ has been plotted for random and scale free networks in Fig. 5 (a) and (c) respectively. Fraction of $u, b$ and $i$ in the neighborhood of a node of different degrees has also been plotted for both the networks in Fig. 5 (b) and (d) respectively. A node with higher degree has higher probability to be in broadcaster class, in random as well as scale free network. For random network, the fraction of believers around a node is independent of its nodal degree, but for scale free network this fraction is not identical. It is again due to the heterogeneous structure of the network and presence of hubs in the network.

\subsubsection{Simulation over Real Networks}

The important parameters associated with some real networks (Hamster network, Email network and Jazz network) collected from KONECT database ${ }^{37}$ are tabulated in Table 1. We have used these networks for our simulation studies; the steady state fractions of unaware $\left(u^{*}\right)$, broadcaster $\left(b^{*}\right)$ and inert $\left(i^{*}\right)$ for various networks are mentioned in Table 2; for comparison, results for homogeneous dynamics are also mentioned in that table.

Though the networks taken from KONECT database account for social connections, and each of them depicts contacts between people in a community, none of these networks are actually recommendation network. To better understand the referral flow, we perform an extensive experiment (see in Sec. 5) to build up a recommendation network. After generating the recommendation network, we observe the final steady states of the network for different parameters and initialization as shown in Fig. 6, where we have shown three different cases. In Fig. 6(a), we set the parameters such that $\mathscr{R}<\mathscr{R}_{c}<1$. In this case, even though we 


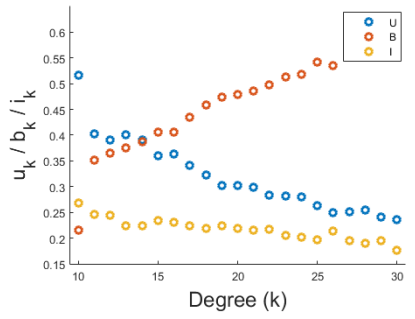

(a)

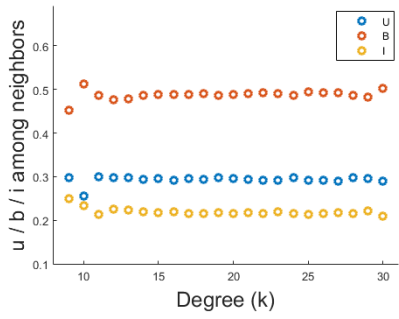

(b)

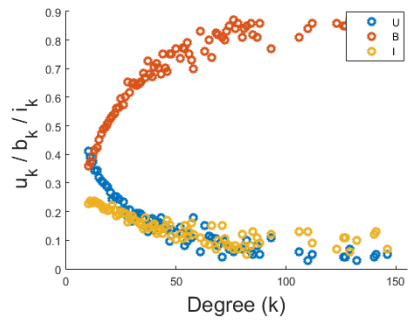

(c)

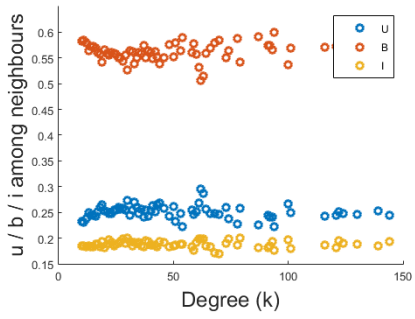

(d)

Figure 5. (a) $u_{k}, b_{k}$ and $i_{k}$ with respect to $k$ at steady-state in random network; (b) Fraction of $u, b$ and $i$ in the neighborhood of a node with degree $k$ in random network; (c) $u_{k}, b_{k}$ and $i_{k}$ with respect to $k$ at steady-state in scale-free network; (d) Fraction of $u, b$ and $i$ in the neighborhood of a node with degree $k$ in scale-free network.

Table 2. Comparison of steady state fractions in various networks, with the homogeneous dynamics $(\mathscr{R}=0.64)$

\begin{tabular}{|c|l|l|l|l|l|l|l|}
\hline $\begin{array}{c}\text { Steady state } \\
\text { fraction }\end{array}$ & $\begin{array}{c}\text { Homogeneous } \\
\text { Setting }\end{array}$ & $\begin{array}{c}\text { Random } \\
\text { Network }\end{array}$ & $\begin{array}{c}\text { Scale-free } \\
\text { Network }\end{array}$ & $\begin{array}{c}\text { Hamster } \\
\text { Network }\end{array}$ & $\begin{array}{c}\text { Email } \\
\text { Network }\end{array}$ & $\begin{array}{c}\text { Jazz } \\
\text { Network }\end{array}$ & $\begin{array}{c}\text { Recommendation } \\
\text { Network }\end{array}$ \\
\hline$u^{*}$ & 0.277 & 0.288 & 0.319 & 0.512 & 0.394 & 0.348 & 0.352 \\
\hline$b^{*}$ & 0.521 & 0.510 & 0.467 & 0.342 & 0.417 & 0.469 & 0.441 \\
\hline$i^{*}$ & 0.202 & 0.202 & 0.214 & 0.146 & 0.189 & 0.183 & 0.207 \\
\hline
\end{tabular}

have 500 broadcasters initially (denoted by $B_{\text {in }}$ in the figure), in the final steady-state there is no broadcaster left. For Figs. 6 (b) and (c), we keep the parameters such that $\mathscr{R}_{c}<\mathscr{R}<1$, i.e., the parameter set is in the region of bistability. In this parameter setting, if we keep $B_{\text {in }}$ very low, the steady state becomes broadcaster free, however, if we start with sufficiently high number of broadcasters, we get endemic steady state even if $\mathscr{R}<1$. Here, Figs. 6 (b) and (c) can be associated with the lower branch (cyan or red) and the upper branch (green or blue) of Fig. 3 (a). The lower branch and the upper branch is detected as mentioned in ${ }^{38}$. In the bistable region, different final states of the recommendation network for different initial conditions exhibits the presence of hysteresis. We also observe that, system's propensity for the endemic state increases as $\mathscr{R}$ goes close to 1 . For different real networks, we consider parameters equivalent to Fig. 3(a) (bottom) with $\mathscr{R}=0.64$ to observe the flow, and the final steady state fractions of $u^{*}, b^{*}$ and $i^{*}$ are compared in Table 2. It can be observed that even when $\mathscr{R}<1,41-51 \%$ of the population belong to the broadcaster class, indicating the survival of the campaign in steady state because of the bistable property.

\section{Summary \& Discussions}

For a successful business, marketing is not only a supportive component, but it is one of the key ingredients ${ }^{39}$. In today's digital environment, the goal of a marketing campaign might have grown beyond enticing a consumer to click on a product icon. Now the objective has expanded to reach maximum number of people and create 'sustained engagement' with the consumer ${ }^{40}$. Weber has correctly pointed out ${ }^{41}$ that to succeed in social media marketing, marketers must 'talk with' the customers, rather than 'talk $a t$ ' them. Surely, a VM campaign provides an inexpensive, personal-level way to reach the customers, where the inherent ignorance or indifference of the customers can be handled well. But with more than 3 million active advertisers posting their ads on social networking sites like Facebook, to reach a bigger audience, it is essential that the campaign establishes substantially long iterations in the population. In last couple of years, marketers have gradually started to use retargeting to convert customers who had formerly window-shopped in their websites, or abandoned carts suddenly ${ }^{33,42-44}$. It has become a familiar part of our web-experience now-a-days to see hyperlinked photos of products that we have once browsed. Using Facebook code snippet called pixel, services of firms like Retargeter, Adroll, Perfect Audience, etc. or using giant exchanges like Google, Twitter ${ }^{45}$, the campaigners are re-engaging lost customers frequently. But some recent works have demonstrated that severe privacy concern and cynicism among consumers is a straightforward effect of constant retargeting, which directly affects the buying intent ${ }^{17,23,24,46}$.

In this paper, we show that, while one way for ensuring re-engagement could be repetitive posting of the campaign by the firm itself, a much more effective way could be creating a framework where some people in the population always remain enthusiastic about the product, and act as ambassadors themselves. As a group of friends, families or colleagues often have similar interests as well as proximity, a social-circle-driven retargeting technique will have much more prospect than conventional company-acted reminder ads. Assuring the unresponsive inert customers about the authenticity, security and 


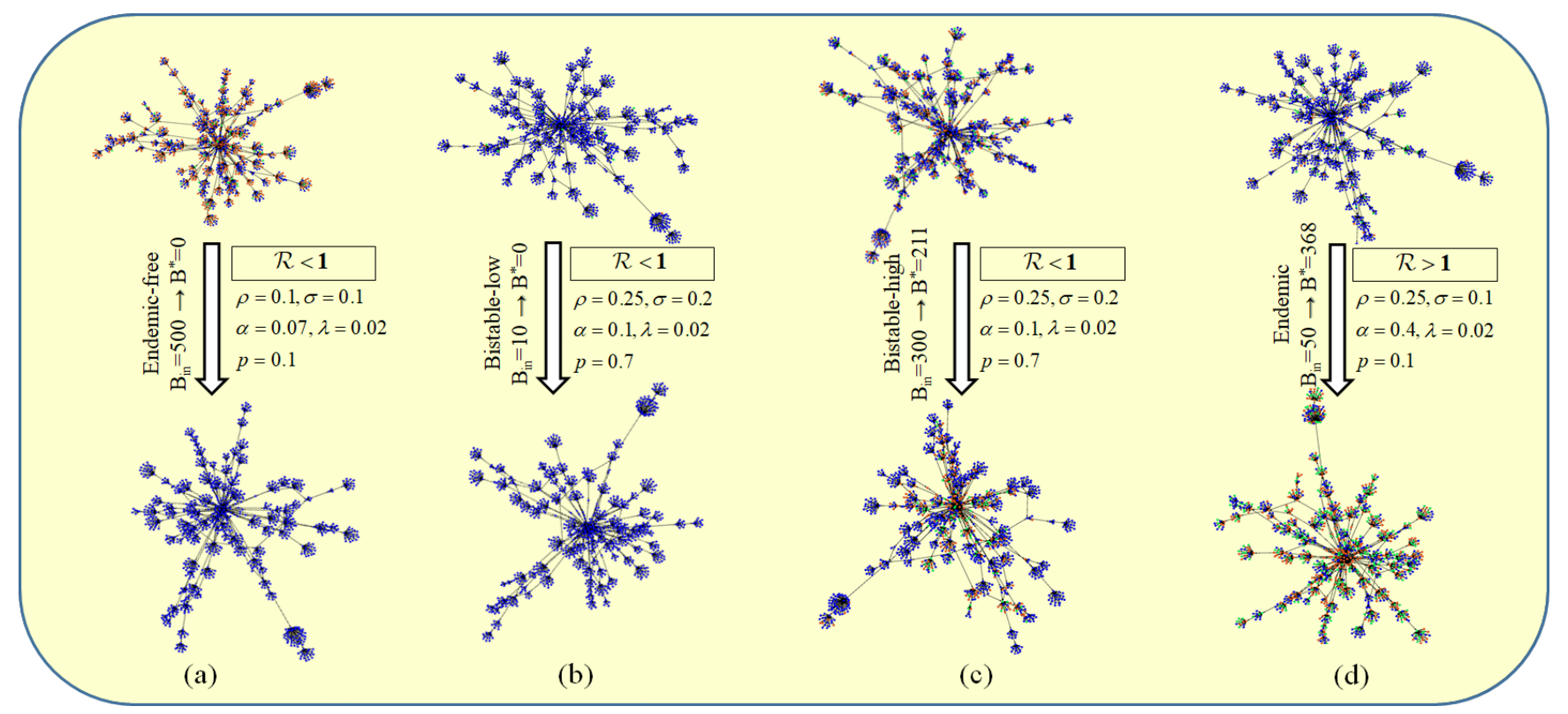

Figure 6. Different steady state transitions of the recommendation network. The top row indicates initial conditions of the network with different initalizations and parameter values, and the bottom row indicates the final state of the network for the corresponding cases. Blue color indicates unaware nodes whereas orange and green indicate broadcaster and inert nodes. $B_{\text {in }}$ and $B *$ indicate initial and final number of broadcasters. (a) When the parameters are selected from an endemic-free region, in the steady state, the population is always broadcaster free. In (b) and (c) parameters are selected from bistable region. Thus, when the network is initialized with small number of broadcaster it reaches to a broadcaster free state, but when initialized with more number of broadcasters, the final steady state is endemic even though $\mathscr{R}<1$. (d) The parameters are selected from an endemic region. So, even the initial number of broadcasters are small, the network always reaches to an endemic steady state.

usefulness of the campaign becomes much more plausible through this way. Our proposed model relies thoroughly on a survey data that deals with consumer mindset and outlook ${ }^{14}$, to prescribe ways to achieve sustainability for online campaigns. We demonstrate that customers have an individuality which cannot be ignored, and inherent aversion towards marketing messages among a considerable percentage of people have to be considered. Beside the creative aspects of the campaign, the brand loyalty and brand name also plays a very important role to create positive reaction of a person towards a campaign message. Exhaustive investigation of our differential equation based quantitative model shows the nonlinear relapse rate, $\alpha$, plays a key role to safeguard the survival of the campaign. We also show that bistability, which comes into picture due to the inherent nonlinearity of the dynamics, works as an opportunity for the firm to make the viral campaign maintain its endemic state, even in adversarial conditions. We have calculated and discussed the critical parameters that help to sustain the desired endemic state by exploiting the properties of bistability.

Exploring beyond the homogeneous system dynamics, we studied the system considering the network structures. Along with model networks, we simulated the dynamics on existing social networks as well as on our experimentally generated recommendation network, to show that regaining those who are not showing any interest despite being aware of the campaign, is extremely important; acquirement, retargeting, and win-back of these customers create the path towards the success of an online marketing campaign. We have also shown that over-usage of advertising mails are many a time treated equivalent to spamming, and create negative brand value instead of a positive impact. For newer brands (i.e., where the value of brand trust parameter $p$ is not in favor), along with the lucrativeness of the offer, figuring out innovative ways to remind the unresponsive customers can be the key to success. With no brand history, these points must be included in their referral policy, otherwise an initial adverse acceptability of the brand might lead to the failure of the entire campaign process.

Our work is the first of its kind, where we introduce NLP based topic modeling to understand customer psychology towards VM campaigns $^{14,15,17,22-24,30-33}$ and implement it in form of a precise dynamical model which mimics epidemic-like behavior. We explore the conditions of success for the campaign in terms of realistic parameters both for homogeneous and heterogeneous population structure. We must mention that from the perspective of an advertising firm, working on a marketing or political campaign, the astonishing effect of the relapse rate and the brand trust parameter can have much deeper consequences. Researchers have already seen evidence that when a relevant win-back offer is given, the once-lost temporarily inert customers become more profitable in their second lifetime ${ }^{47}$. Our findings firmly establishes several recent observations ${ }^{41,47}$ on quantitative grounds that rebuilding a targeted buzz in market can many times have a much stronger effect than the initial buzz itself. Not 
only for marketing, but also for remarketing, the companies should not act as broadcasters themselves, but instead, they should channelize their message through personal and social influences.

Beyond the findings we report in this paper, our methodology has potential to push forward the field of quantitative modelling of referral marketing dynamics. Firstly, this brand-trust parameter $p$ brings the idea of realistic decision making to quantitatively understand referral marketing psychology. This parameter is chosen to give an estimate of the perceived trust the community has for a particular brand, so that, without any hesitation, they can participate in a marketing campaign. With this idea of perceived trust, our referral marketing model can also be studied from the perspective of evolutionary game theory. In voluntary participation to a marketing campaign, an individual's decision-making may depend on several factors such as the trust towards the brand, consequences after participation, incentives associated with a campaign and fear of phishing or spamming. In a future study, we will proceed to associate the dependence of decision making on the behavioural response of fellow community members, and model the problem from the point-of-view of the traditional social dilemma depicted by game theory. Considering the balance between perceived trust towards the brand, and perceived risk associated with referral marketing campaigns, an effective pay-off could be calculated, and thus, Nash equilibrium could be detected, which will have striking similarities with vaccination game dynamics ${ }^{48}$. This work is the first step which can connect the well-developed tools of vaccination games with the strategies of referral marketing policies causing a huge benefit for the marketing community.

Another highlight of our proposed methodology is that it is extremely promising in bridging the gap between social surveys and mathematical modelling, in general. Inside and outside the boundaries of marketing field, several social issues involving public psychology are commonly explored and researched by committed efforts of survey-based studies. On the other hand, mathematical models and computation based analysis have also shown great implications in understanding and predicting complex dynamical social systems. To our knowledge, there is no other study where a concrete methodology has been proposed with survey-based data giving direct inputs to model construction, not by heuristic arguments but by solid quantitative study through extensive language processing. Combining open-ended survey responses, NLP based tools, mean-field study and network analysis, we attempted to put the pieces of a puzzle together which can offer a very general comprehensive methodology to mathematically and computationally analyse survey responses, make concrete predictions on behavioural reaction through mathematical models, and see the implications in real-life social networks.

\section{Methods}

\subsection{Analyses of Survey Data}

On the survey data of Ghosh et al. ${ }^{14}$, first we perform a series of preprocessing, which includes removal of English stop words, punctuation and numbers ${ }^{49}$ from the data, the lower-case conversion and lemmatization to have a standard representation of the text. The first level analysis was performed through term frequency ${ }^{19}$ analysis to detect frequently used words by the users to answer their questions. To better understand the correlation between these words, next, we perform topic modeling on the processed text data. As the responses are longer, instead of using LFLDA ${ }^{50}, \mathrm{BTM}^{51}$ or WNTM ${ }^{52}$, we use classical $\mathrm{LDA}^{20}$ to find out the topics in the data. The optimal number of topics in the LDA model is decided using the coherence score.

\subsection{Mean Field Study: Equilibrium Analysis}

The system of Eq. 1 can have two kinds of equilibrium: a VM-free equilibrium $E_{0}$ (with the entire population being unaware), and an Endemic equilibrium $E^{\star}$ (with a finite percentage of broadcasters present in steady state). As there is no time evolution at equilibrium, all the components of $E^{\star}$ can be evaluated by equating $u^{\prime}, b^{\prime}$ and $i^{\prime}$ of Eq. 1 to zero. While solving for $E^{\star}$, the first equation of system model, defined in Eq. 1, gives

$$
u^{\star}=\frac{\mu}{\rho b^{\star}+\mu}
$$

Relevant substitutions from Eq. 13 and replacing $i^{\star}$ by $\left(1-b^{\star}-u^{\star}\right)$, simple algebra results into $l\left(b^{\star}\right)^{2}+m b^{\star}+n=0$, where

$$
\begin{aligned}
l & =\alpha p \rho \\
m & =(\sigma \rho+\mu \rho+\lambda \rho+\alpha p \mu-\alpha p \rho) \\
n & =\mu(\sigma+\mu+\lambda)-\rho(\lambda+\mu p) .
\end{aligned}
$$

Examining the coefficients, we conclude that $l$ is always positive; $m$ is positive for small values of $\alpha$, and $n$ is positive or negative depending on whether $\frac{\rho(\lambda+\mu p)}{\mu(\lambda+\mu+\sigma)}=\mathscr{R}$ is smaller or greater than 1 . Two completely different steady state scenarios can arise:

Case 1: For negative $n$ (i.e., $\mathscr{R}>1$ ), the quadratic equation has a unique positive solution $b_{+}^{\star}$, as another solution $b_{-}^{\star}$ is always negative and so, unphysical, and there exists a unique endemic equilibrium $E^{\star}$ whenever $\mathscr{R}>1$.

Case 2: On the other hand, for positive $n$ (i.e., $\mathscr{R}<1$ ), the number of physical roots of the equation depends on the sign of $q$, and therefore, the nonlinear relapse parameter $\alpha$. Depending on this fact if $\alpha$ is high (or low), multiple (or no) endemic equilibria may exist.

For analysing stability of these equilibria, we consider Eq. 2 as

$$
\begin{aligned}
& f_{1}=\mu-\rho b u-\mu u \\
& f_{2}=p \rho b u+\lambda i+\alpha p b i-\sigma b-\mu b \\
& f_{3}=\sigma b+(1-p) \rho b u-\lambda i-\alpha p b i-\mu i
\end{aligned}
$$


and calculate the Jacobian of $\mathbf{f}$, where $\mathbf{f}=\left[f_{1}, f_{2}, f_{3}\right]^{13}$.

\subsection{Parameter selection in Network}

Instead of same $\rho$ and $\alpha$ for every node, as considered in the mean-field analysis, the rates have been made proportional to degree of the nodes. $\rho_{n}$ and $\alpha_{n}$ are chosen to be $\frac{\rho}{\langle k\rangle}$ and $\frac{\alpha}{\langle k\rangle}$ respectively so that expected values of $\rho_{n} k$ and $\alpha_{n} k$ are $\rho$ and $\alpha$ used in mean-field analysis. It synchronizes the parameter set of both the approaches and allows us to compare the obtained results.

\subsection{Initial Propagation in Network}

In initial phase of message spreading ${ }^{53}, b$ and $i$ can be approximated by zero and $u$ by 1 . Using these values in Eq. 8, we get

$$
\begin{aligned}
\Theta_{u}^{\prime} & =\mu-\rho_{n} \frac{\left\langle k^{2}\right\rangle}{\langle k\rangle} \Theta_{b}-\mu \Theta_{u} \\
\Theta_{b}^{\prime} & =p \rho_{n} \frac{\left\langle k^{2}\right\rangle}{\langle k\rangle} \Theta_{b}+\lambda \Theta_{i}-(\sigma+\mu) \Theta_{b} \\
\Theta_{i}^{\prime} & =\sigma \Theta_{b}+(1-p) \rho_{n} \frac{\left\langle k^{2}\right\rangle}{\langle k\rangle} \Theta_{b}-(\lambda+\mu) \Theta_{i}
\end{aligned}
$$

Last two equations of the Eq. 16 forms a system of simultaneous linear differential equations with constant coefficients.

$$
\begin{aligned}
\frac{\mathrm{d} \Theta_{b}}{\mathrm{~d} t} & =C_{1} \Theta_{b}+C_{2} \Theta_{a} \\
\frac{\mathrm{d} \Theta_{a}}{\mathrm{~d} t} & =C_{3} \Theta_{b}+C_{4} \Theta_{a}
\end{aligned}
$$

\subsection{Steady State Condition in Network Propagation}

In large time limit, system will reach steady state. Rate of change of fractions $u, b$ and $i$ will be zero. In case of degree based compartment scheme, $u_{k}, b_{k}$ and $i_{k}$ will not change. Equating first and third equation of Eq. 7 to zero, we have

$$
u_{k}=\frac{\mu}{\mu+\rho_{n} k \Theta_{b}} \quad \text { and } \quad i_{k}=\frac{\sigma b_{k}+(1-p) \rho_{n} k u_{k} \Theta_{b}}{\lambda+\mu+\alpha_{n} k p \Theta_{b}}
$$

Putting these values in second equation of the same set will give

$$
b_{k}=\frac{\rho_{n} k \Theta_{b}\left(\mu p+\lambda+\alpha_{n} k p \Theta_{b}\right)}{\left(\mu+\rho_{n} k \Theta_{b}\right)\left(\lambda+\mu+\sigma+\alpha_{n} k p \Theta_{b}\right)}
$$

Multiplying $b_{k}$ by $\frac{k p_{k}}{\langle k\rangle}$ and performing summation over $k$, we get

$$
\Theta_{b}=\frac{1}{\langle k\rangle} \sum_{k} \frac{p_{k} k^{2} \rho_{n} \Theta_{b}\left(\mu p+\lambda+\alpha_{n} k p \Theta_{b}\right)}{\left(\mu+\rho_{n} k \Theta_{b}\right)\left(\lambda+\mu+\sigma+\alpha_{n} k p \Theta_{b}\right)}
$$

This above expression is a self consistency equation of $\Theta_{b}$, i.e. Eq. 21 can be written as $\Theta_{b}=f\left(\Theta_{b}\right)$.

\subsection{Simulation over Networks}

The simulations are performed over random network and scale-free network having 1024 nodes and average degree 10. Our random network follows Erdös-Rényi model with binomial degree distribution which converges to Poisson distribution if the network has very large number of nodes. The scale-free network that we have used for our analyses is generated using Barabási-Albert model of preferential attachment ${ }^{54}$ and it follows power law degree distribution having power exponent 3. Though, both random network and scale-free network are popular in network science, most of the real world networks do not follow the characteristics of any particular network model. Thus, we have simulated the flow of recommendation campaigns under different conditions over several real world networks. These analyses help us to understand the behavior in real social interaction scenarios. For our simulation studies over real networks, we collect some popular social networks from KONECT database ${ }^{37}$, namely, Hamster network, Email network and Jazz network. But, as these networks are not generated from any recommendation campaign, we have also generated a recommendation network from a referral experiment.

\subsection{Experiment for Generation of Recommendation Network}

We have selected 5 people who know each other and asked them to forward a mail with a make-believe referral marketing message. The policy for the recommendation is as follows: after forwarding the message to his contacts, a person will get credit points depending on the number of forwards his/her contacts made. For example, if Alice forwards the message to 10 friends, and out of 10, 4 persons forward the message further to some other people, then Alice wins 4 credit points. To track the propagation of the message and to the network, we have asked everyone to send one copy of the forwarded message to our groups' email address, so that we can draw the connections between the participants. Two friends of Alice may send the message to each other even if both of them receive the message from Alice. Though, in our experiment, the network structure becomes a directed graph, but we assume that the network structure is undirected, because if ' $\mathrm{A}$ ' can send a viral message to 'B', 'B' may also send some other viral message to 'A'. As the experiment goes on, we also receive some isolated points and branches. The isolated branches may arise if someone forwards the message to other people but forgets to include our email id while forwarding the message, and his/her contacts forward the message following the instructions correctly. For the ease of discussion, we have removed the isolated branches while building the final network. Finally, we have a sparse network with 1157 nodes and 2558 edges. 


\section{Data Availability}

All data are available on request.

\section{References}

1. Holmes, E. E., Lewis, M. A., Banks, J. \& Veit, R. Partial differential equations in ecology: spatial interactions and population dynamics. Ecology 75, 17-29 (1994).

2. Gaurav, K., Ghosh, S., Bhattacharya, S. \& Singh, Y. N. Equilibria of rumor propagation: Deterministic and network approaches. In Region 10 Conference, IEEE TENCON 2017, 2029-2034 (IEEE, 2017).

3. Woo, J., Son, J. \& Chen, H. An sir model for violent topic diffusion in social media. In Intelligence and Security Informatics (ISI), 2011 IEEE International Conference on, 15-19 (IEEE, 2011).

4. Shtatland, E. S. \& Shtatland, T. Early detection of epidemic outbreaks and financial bubbles using autoregressive models with structural changes. Proc. NESUG 21 (2008).

5. Kermack, W. O. \& McKendrick, A. G. Contributions to the mathematical theory of epidemics. ii.- the problem of endemicity. Proc. R. Soc. Lond. A 138, 55-83 (1932).

6. Fan, D. P. Ideodynamics: The kinetics of the evolution of ideas. J. Math. Sociol. 11, 1-23 (1985).

7. Diekmann, O., Heesterbeek, J. A. P. \& Metz, J. A. On the definition and the computation of the basic reproduction ratio $\mathrm{R}_{0}$ in models for infectious diseases in heterogeneous populations. J. mathematical biology 28, 365-382 (1990).

8. Blackmore, S. The meme machine (vol. 25) (2000).

9. Bobashev, G. V., Goedecke, D. M., Yu, F. \& Epstein, J. M. A hybrid epidemic model: combining the advantages of agent-based and equation-based approaches. In Simulation Conference, 2007 Winter, 1532-1537 (IEEE, 2007).

10. Bass, F. M. A new product growth for model consumer durables. Manag. science 15, 215-227 (1969).

11. Sohn, K., Gardner, J. T. \& Weaver, J. L. Viral marketing-more than a buzzword. J. Appl. Bus. Econ. 14, 21-42 (2013).

12. Rodrigues, H. S. \& Fonseca, M. J. Can information be spread as a virus? viral marketing as epidemiological model. Math. Methods Appl. Sci. 39, 4780-4786 (2016).

13. Bhattacharya, S., Gaurav, K. \& Ghosh, S. Viral marketing on social networks: An epidemiological perspective. Phys. A: Stat. Mech. its Appl. 525, 478-490 (2019).

14. Ghosh, S., Bhattacharya, S., Gaurav, K. \& Singh, Y. N. Going viral: The epidemiological strategy of referral marketing. arXiv preprint arXiv:1808.03780 (2018).

15. Raad, M., Yeassen, N. M., Alam, G. M., Zaidan, B. \& Zaidan, A. Impact of spam advertisement through e-mail: A study to assess the influence of the anti-spam on the e-mail marketing. Afr. J. Bus. Manag. 4, 2362-2367 (2010).

16. Jayawardhena, C., Kuckertz, A., Karjaluoto, H. \& Kautonen, T. Antecedents to permission based mobile marketing: an initial examination. Eur. journal marketing 43, 473-499 (2009).

17. Morimoto, M. \& Chang, S. Consumers' attitudes toward unsolicited commercial e-mail and postal direct mail marketing methods: intrusiveness, perceived loss of control, and irritation. J. Interact. Advert. 7, 1-11 (2006).

18. Tashakkori, A. \& Teddlie, C. Mixed methodology: Combining qualitative and quantitative approaches, vol. 46 (Sage, 1998).

19. Jotikabukkana, P., Sornlertlamvanich, V., Manabu, O. \& Haruechaiyasak, C. Effectiveness of social media text classification by utilizing the online news category. In 2015 2nd International Conference on Advanced Informatics: Concepts, Theory and Applications (ICAICTA), 1-5 (IEEE, 2015).

20. Blei, D. M., Ng, A. Y. \& Jordan, M. I. Latent dirichlet allocation. J. machine Learn. research 3, 993-1022 (2003).

21. Zhao, Z. \& Renard, D. Viral promotional advergames: how intrinsic playfulness and the extrinsic value of prizes elicit behavioral responses. J. Interact. Mark. 41, 94-103 (2018).

22. Phelps, J. E., Lewis, R., Mobilio, L., Perry, D. \& Raman, N. Viral marketing or electronic word-of-mouth advertising: Examining consumer responses and motivations to pass along email. J. advertising research 44, 333-348 (2004).

23. Leppaniemi, M. \& Karjaluoto, H. Factors influencing consumers' willingness to accept mobile advertising: a conceptual model. Int. J. Mob. Commun. 3, 197-213 (2005).

24. Barnes, S. J. \& Scornavacca, E. Mobile marketing: the role of permission and acceptance. Int. J. Mob. Commun. 2, 128-139 (2004).

25. Chaudhuri, A. \& Holbrook, M. B. The chain of effects from brand trust and brand affect to brand performance: the role of brand loyalty. J. marketing 65, 81-93 (2001).

26. D. Hollebeek, L. \& Chen, T. Exploring positively-versus negatively-valenced brand engagement: a conceptual model. J. Prod. \& Brand Manag. 23, 62-74 (2014). 
27. Fournier, S. \& Alvarez, C. Brands as relationship partners: Warmth, competence, and in-between. J. Consumer Psychol. 22, 177-185 (2012).

28. Chu, S.-C. Viral advertising in social media: Participation in facebook groups and responses among college-aged users. J. interactive advertising 12, 30-43 (2011).

29. Briggs, T. Social media's second act: toward sustainable brand engagement. Des. Manag. Rev. 21, 46-53 (2010).

30. Ellison, N. B., Steinfield, C. \& Lampe, C. The benefits of facebook “friends:” social capital and college students' use of online social network sites. J. computer-mediated communication 12, 1143-1168 (2007).

31. https://www.marketingsherpa.com/article/chart/why-consumers-unsubscribe. Online (Accessed: 30.08.2019).

32. Silva, K. https://www.business2community.com/email-marketing/surprising-truth-sending-many-emails-01786693. Online (Accessed: 30.08.2019).

33. Berke, A., Fulton, G. \& Vaccarello, L. The retargeting playbook: How to turn web-window shoppers into customers (John Wiley \& Sons, 2014).

34. Loewenstein, G. Out of control: Visceral influences on behavior. Organ. behavior human decision processes 65, 272-292 (1996).

35. Wolny, J. \& Mueller, C. Analysis of fashion consumers' motives to engage in electronic word-of-mouth communication through social media platforms. J. marketing management 29, 562-583 (2013).

36. Strogatz, S. H. Nonlinear dynamics and chaos: with applications to physics, biology, chemistry, and engineering (CRC Press, 2018).

37. Kunegis, J. Konect: The koblenz network collection. In Proceedings of the 22nd International Conference on World Wide Web, 1343-1350 (ACM, 2013).

38. Tunc, I. \& Shaw, L. B. Effects of community structure on epidemic spread in an adaptive network. Phys. Rev. E 90, 022801 (2014).

39. Drucker, P. The society of organizations. Harv. business review 95104 (1992).

40. Harris, L. \& Rae, A. Social networks: the future of marketing for small business. J. business strategy 30, 24-31 (2009).

41. Weber, L. Marketing to the social web: How digital customer communities build your business (John Wiley \& Sons, 2009).

42. Ismail, Z. \& Kolbegger, C. Correlated consumer telephone numbers and user identifiers for advertising retargeting (2014). US Patent App. 13/865,966.

43. Moriguchi, T., Xiong, G. \& Luo, X. Retargeting ads for shopping cart recovery: Evidence from online field experiments. Available at SSRN 2847631 (2016).

44. Yeo, J., Kim, S., Koh, E., Hwang, S.-w. \& Lipka, N. Predicting online purchase conversion for retargeting. In Proceedings of the Tenth ACM international conference on web search and data mining, 591-600 (ACM, 2017).

45. Finkle, T. A. Adroll: A case study of entrepreneurial growth. New Engl. J. Entrepreneurship 16, 47-50 (2013).

46. Zarouali, B., Ponnet, K., Walrave, M. \& Poels, K. Do you like cookies? Adolescents' skeptical processing of retargeted facebook-ads and the moderating role of privacy concern and a textual debriefing. Comput. Hum. Behav. 69, 157-165 (2017).

47. Kumar, V., Bhagwat, Y. \& Zhang, X. Regaining "lost" customers: The predictive power of first-lifetime behavior, the reason for defection, and the nature of the win-back offer. J. Mark. 79, 34-55 (2015).

48. Bauch, C. T. \& Earn, D. J. Vaccination and the theory of games. Proc. Natl. Acad. Sci. 101, 13391-13394 (2004).

49. Pietsch, A.-S. \& Lessmann, S. Topic modeling for analyzing open-ended survey responses. J. Bus. Anal. 1, 93-116 (2018).

50. Nguyen, D. Q., Billingsley, R., Du, L. \& Johnson, M. Improving topic models with latent feature word representations. Transactions Assoc. for Comput. Linguist. 3, 299-313 (2015).

51. Yan, X., Guo, J., Lan, Y. \& Cheng, X. A biterm topic model for short texts. In Proceedings of the 22nd international conference on World Wide Web, 1445-1456 (ACM, 2013).

52. Zuo, Y., Zhao, J. \& Xu, K. Word network topic model: a simple but general solution for short and imbalanced texts. Knowl. Inf. Syst. 48, 379-398 (2016).

53. Liu, J., Tang, Y. \& Yang, Z. The spread of disease with birth and death on networks. J. Stat. Mech. Theory Exp. 2004, P08008 (2004).

54. Barabási, A.-L. \& Albert, R. Emergence of scaling in random networks. Science 286, 509-512 (1999).

\section{Author contributions statement}

S.B. and S.G. conceived the idea of the study. K.G. and S.G. performed the simulations and the experiments. S.G. and K.G. analysed the results. Y.N.S., S.B. and S.G. prepared the manuscript. All authors reviewed the manuscript. 